\title{
current events
}

This section carries events of interest to the synchrotron radiation community. Works intended for this section should be sent direct to the Current-Events Editor (s.hasnain@dl.ac.uk).

\section{Chancellor Schröder starts user operation of the VUV-FEL at DESY}

On 3 August 2005, German Federal Chancellor Gerhard Schröder opened the new free-electron laser VUV-FEL at DESY, formally opening it to the user community. 'The VUV-FEL at DESY is the worldwide first free-electron laser for the short-wavelength range of ultraviolet radiation. It generates especially intense and extremely short flashes of laser light that open up completely new insights into the nanoworld', said DESY Research Director Professor Jochen Schneider. "Using the VUV-FEL, scientists can for instance 'film' chemical reactions. The unique radiation enables ground-breaking experiments in fields such as cluster physics, solid-state physics, surface physics, plasma research and molecular biology."

At present, a total of 29 research projects are planned at the VUVFEL with the scientific community from over 50 institutes originating from 11 countries. The construction cost of the VUV-FEL project was 117 million Euros (US $\$ 160 \mathrm{M}$ ), $90 \%$ of which came from public funds (of which $90 \%$ came from the Federal Republic of Germany and 10\% from the City of Hamburg) and 10\% from international partners.

The DESY VUV-FEL is tunable within the wavelength range from 6 to $30 \mathrm{~nm}$ ( 60 to $300 \AA$ ). The extremely short duration of its very intense radiation pulses, which last only 10 to $50 \mathrm{fs}$, is especially important. It allows scientists for example to directly observe the formation of chemical bonds. The very high power density of radiation would allow scientists to create energy densities in matter in the laboratory which are so high that they can normally only be found in the cosmos, a topic of major interest to plasma physics.

The free-electron laser VUV-FEL makes use of the new technology which was developed at DESY from 1992 to 2004 by the international team of the TESLA Collaboration. In a first step, electrons are brought to high energies by a superconducting linear accelerator. They then race through an undulator, which forces them to follow a slalom course and thereby radiate flashes of light. According to the SASE principle of 'self-amplified spontaneous emission', the process finally generates the short-wavelength intense

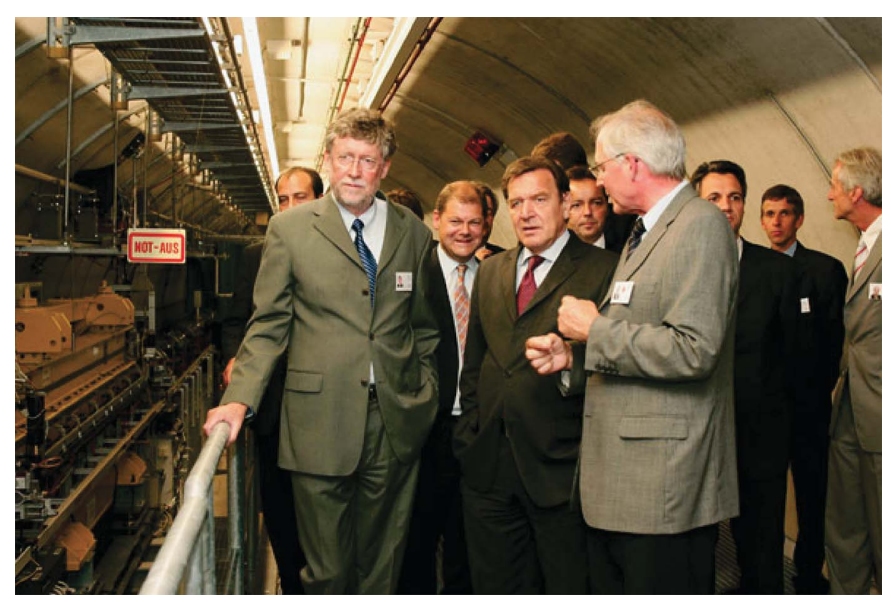

Professor Jochen Schneider (right) explains the capabilities of DESY VUVFEL to Chancellor Gerhard Schröder. In the background a section of the VUV-FEL undulator can be seen. flashes of laser light. As a user facility, the DESY VUV-FEL will offer a total of five experimental stations, at which different instruments can be operated alternately.

The operation of DESY VUV-FEL is expected to provide important insights for the planned $3.4 \mathrm{~km}$-long European X-ray laser XFEL. The XFEL is expected to generate even shorter wavelengths down to $0.085 \mathrm{~nm}(0.8 \AA)$ and is expected to follow the US and Japanese X-ray free-electron laser facility (LCLS at Stanford and SCSS in Harima) in the early half of the next decade [J. Synchrotron Rad. (2005). 12, 386-388; 543-544].

\section{US Protein Structure Initiative advances to rapid production phase}

With the announcement of ten new research centers, the Protein Structure Initiative (PSI) launched the second phase of its national effort for high-throughput structure determination for a wide range of proteins. Selection of the centers, slated to receive about USD $\$ 300$ million over the next five years, marks the second half of the decadelong initiative funded largely by the National Institute of General Medical Sciences (NIGMS), part of the National Institutes of Health.

When the PSI established its pilot centers beginning in 2000, its goal was twofold: to develop innovative approaches and tools, such as robotic instruments, that streamline and speed up many steps of generating protein structures; and to incorporate those new methods into pipelines that turn DNA sequence information into protein structures.

Now, the focus shifts to a production phase during which the new centers will use methods developed during the pilot period to rapidly determine thousands of protein structures found in organisms ranging from bacteria to humans. These efforts will facilitate structure determination on a much larger number of proteins through computer modelling.

The PSI production phase includes two types of centers. Four large-scale centers, established during the pilot phase, expect to generate between 3000 and 4000 structures. Six specialized centers will develop novel methods for quickly determining the structures of proteins that traditionally have been difficult to study. These include small protein complexes; proteins that attach to a cell's outer envelope, or membrane; and many proteins from higher organisms, including humans.

The large-scale centers are:

Joint Center for Structural Genomics (led by Ian Wilson, of the Scripps Research Institute in La Jolla, CA);

Midwest Center for Structural Genomics (led by Andrzej Joachimiak, of the Argonne National Laboratory near Chicago, IL);

New York Structural GenomiX Research Consortium (led by Stephen Burley, of Structural GenomiX in San Diego, CA);

Northeast Structural Genomics Consortium (led by Gaetano Montelione, of Rutgers University in New Brunswick, NJ).

These centers had produced the bulk of structures in phase 1 of the PSI projects and were the top four producers of novel as well as unique structures. Thus, on 2 August, data released by phase 1 centers had recorded 179 (Wilson), 262 (Joachimiak), 147 (Burley) and 98 (Montelione) novel structures. 
The specialized centers are:

Accelerated Technologies Center for Gene to 3D Structure (led by Lance Stewart, of deCODE biostructures, Bainbridge Island, WA);

Center for Eukaryotic Structural Genomics (led by John Markley, of the University of Wisconsin-Madison);

Center for High-Throughput Structural Biology (led by George DeTitta, of Hauptman-Woodward Medical Research Institute, Buffalo, NY);

Center for Structures of Membrane Proteins (led by Robert Stroud, of the University of California, San Francisco, CA);

Integrated Center for Structure and Function Innovation (led by

Thomas Terwilliger, of Los Alamos National Laboratory, NM);

New York Consortium on Membrane Protein Structure (led by Wayne Hendrickson, of New York Structural Biology Center, NY).

\section{Australian synchrotron signs up collaborations agreements with the BSRF, ESRF, SPring-8 and APS}

The Australian Minister for Innovation, John Brumby, has been busy internationalizing the Australian synchrotron radiation effort. On 12 July, he announced the signing up of a new partnership with China's Beijing Synchrotron Radiation Facility (BSRF). A week earlier, a Memorandum of Understanding (MOU) was signed with the European Synchrotron Radiation Facility (ESRF), and in early June he signed an agreement with the world's largest synchrotron facility, SPring-8. The latest agreement has come with the signing of a MOU with the Advanced Photon Source (APS) in Chicago, USA.

This outreach is commendable and will provide easy access to experiences of existing synchrotron light sources. It should also foster international collaboration and encourage the development of new experimental technology, such as new-generation detectors [J. Synchrotron Rad. (2005). 12, 254-255], to improve synchrotron research techniques.

Thus, on signing the agreement with Beijing, the Australian Minister said that 'the Beijing Synchrotron Radiation Facility is a mature synchrotron with a strong track record in delivering science excellence and innovation'. He added, 'Australian researchers will gain access to Chinese experience and expertise, and China will gain access to exciting developments at the Australian synchrotron. This agreement will allow scientists to move freely between the two facilities, exchanging ideas and developing new experimental techniques and technology.' Likewise, on signing a MOU on scientific collaboration with the APS, America's largest and most powerful synchrotron, he said, 'I am delighted APS and the Australian synchrotron have signed a pact to exchange ideas and explore new experimental technology for advanced materials science, life science, earth science, environmental science and industrial research'. $\mathrm{Mr}$ Brumby said, "This agreement means Australia now has science links with all of the world's big three synchrotron facilities". 